\title{
On Lipschitz Perturbations of a Self-Adjoint Strongly Positive Operator
}

\author{
Dinu Teodorescu ${ }^{1}$ and N. Hussain ${ }^{2}$ \\ ${ }^{1}$ Department of Mathematics, Valahia University of Targoviste, Boulevard Unirii 18, 130024 Targoviste, Romania \\ ${ }^{2}$ Department of Mathematics, King Abdulaziz University, P.O. Box 80203, Jeddah 21589, Saudi Arabia
}

Correspondence should be addressed to Dinu Teodorescu; dteodorescu2003@yahoo.com

Received 5 May 2013; Accepted 13 September 2013

Academic Editor: Satit Saejung

Copyright (C) 2013 D. Teodorescu and N. Hussain. This is an open access article distributed under the Creative Commons Attribution License, which permits unrestricted use, distribution, and reproduction in any medium, provided the original work is properly cited.

In this paper we study semilinear equations of the form $A u+\lambda F(u)=f$, where $A$ is a linear self-adjoint operator, satisfying a strong positivity condition, and $F$ is a nonlinear Lipschitz operator. As applications we develop Krasnoselskii and Ky Fan type approximation results for certain pair of maps and to illustrate the usability of the obtained results, the existence of solution of an integral equation is provided.

\section{Introduction and Preliminaries}

The study of abstract operator equations involving linear or nonlinear operators has generated over time useful instruments in the approach of some concrete equations. Therefore, we consider as interesting to present some aspects regarding the semilinear abstract operator equations in Hilbert spaces.

Let $H$ be a real Hilbert space endowed with the inner product $\langle\cdot, \cdot\rangle$ and the norm $\|\cdot\|$.

In $[1,2]$ semilinear equations of the form $A u-F(u)=0$ were studied, where $A: D(A) \subset H \rightarrow H$ is a self-adjoint linear operator with the resolvent set $R(A)$ and $F: H \rightarrow H$ is a Gateaux differentiable gradient operator. If there exist real numbers $a<b$ such that $[a, b] \subset R(A)$ and

$$
a \leq \frac{\langle F(u)-F(v), u-v\rangle}{\|u-v\|^{2}} \leq b,
$$

for all $u, v \in H, u \neq v$ (i.e., $F$ interacts suitably with the spectrum of $A$ ), then it is proved in [2] that the equation $A u-F(u)=0$ has exactly one solution.

The author in [3] presented an existence and uniqueness result for the semilinear equation $A u+F(u)=f$, where $A$ : $D(A) \subset H \rightarrow H$ is a linear maximal monotone operator, satisfying a strong positivity condition, and the nonlinearity $F: H \rightarrow H$ is a Lipschitz operator.
Let $X$ be a real Banach space, ordered by a cone $K$. A cone $K$ is a closed convex subset of $X$ with $\lambda K \subseteq K(\lambda \geq 0)$, and $K \cap(-K)=\{0\}$. As usual $x \leq y \Leftrightarrow y-x \in K$.

Definition 1. Let $M$ be a nonempty subset of an ordered Banach space $X$ with order $\leq$. Two mappings $S, T: M \rightarrow M$ are said to be weakly isotone increasing if $S x \leq T S x$ and $T x \leq S T x$ hold for all $x \in M$. Similarly, we say that $S$ and $T$ are weakly isotone decreasing if $T x \geq S T x$ and $S x \geq T S x$ hold for all $x \in M$. The mappings $S$ and $T$ are said to be weakly isotone if they are either weakly isotone increasing or weakly isotone decreasing.

In our considerations, the following definition will play an important role. Let $\mathscr{B}(X)$ denote the collection of all nonempty bounded subsets of $X$ and $\mathscr{W}(X)$ the subset of $\mathscr{B}(X)$ consisting of all weakly compact subsets of $X$. Also, let $B_{r}$ denote the closed ball centered at 0 with radius $r$.

Definition 2 (see [4]). A function $\psi: \mathscr{B}(X) \rightarrow \mathbb{R}_{+}$is said to be a measure of weak noncompactness if it satisfies the following conditions.

(1) The family $\operatorname{ker}(\psi)=\{M \in \mathscr{B}(X): \psi(M)=0\}$ is nonempty, and $\operatorname{ker}(\psi)$ is contained in the set of relatively weakly compact sets of $X$. 
(2) $M_{1} \subseteq M_{2} \Rightarrow \psi\left(M_{1}\right) \leq \psi\left(M_{2}\right)$.

(3) $\psi(\overline{\mathrm{CO}}(M))=\psi(M)$, where $\overline{\mathrm{CO}}(M)$ is the closed convex hull of $M$.

(4) $\psi\left(\lambda M_{1}+(1-\lambda) M_{2}\right) \leq \lambda \psi\left(M_{1}\right)+(1-\lambda) \psi\left(M_{2}\right)$ for $\lambda \in[0,1]$.

(5) If $\left(M_{n}\right)_{n \geq 1}$ is a sequence of nonempty weakly closed subsets of $X$ with $M_{1}$ bounded and $M_{1} \supseteq M_{2} \supseteq \cdots \supseteq$ $M_{n} \supseteq \cdots$ such that $\lim _{n \rightarrow \infty} \psi\left(M_{n}\right)=0$, then $M_{\infty}:=$ $\bigcap_{n=1}^{\infty} M_{n}$ is nonempty.

The family ker $\psi$ described in (1) is said to be the kernel of the measure of weak noncompactness $\psi$. Note that the intersection set $M_{\infty}$ from (5) belongs to ker $\psi$ since $\psi\left(M_{\infty}\right) \leq$ $\psi\left(M_{n}\right)$ for every $n$, and $\lim _{n \rightarrow \infty} \psi\left(M_{n}\right)=0$. Also, it can be easily verified that the measure $\psi$ satisfies

$$
\psi\left(\overline{M^{w}}\right)=\psi(M)
$$

where $\overline{M^{w}}$ is the weak closure of $M$. if

A measure of weak noncompactness $\psi$ is said to be regular

$$
\psi(M)=0 \text { iff } M \text { is relatively weakly compact, }
$$

subadditive if

$$
\psi\left(M_{1}+M_{2}\right) \leq \psi\left(M_{1}\right)+\psi\left(M_{2}\right),
$$

homogeneous if

$$
\psi(\lambda M)=|\lambda| \psi(M), \quad \lambda \in \mathbb{R},
$$

and set additive (or has the maximum property) if

$$
\psi\left(M_{1} \cup M_{2}\right)=\max \left(\psi\left(M_{1}\right), \psi\left(M_{2}\right)\right) .
$$

The first important example of a measure of weak noncompactness has been defined by de Blasi [5] as follows:

$$
\begin{gathered}
w(M)=\inf \{r>0: \text { there exists } W \in \mathscr{W}(X) \\
\text { with } \left.M \subseteq W+B_{r}\right\},
\end{gathered}
$$

for each $M \in \mathscr{B}(X)$.

Notice that $w(\cdot)$ is regular, homogeneous, subadditive, and set additive (see [5]).

By a measure of noncompactness on a Banach space $X$, we mean a map $\psi: \mathscr{B}(X) \rightarrow \mathbb{R}_{+}$which satisfies conditions (1)-(5) in Definition 2 relative to the strong topology instead of the weak topology.

Definition 3. Let $X$ be a Banach space and $\psi$ a measure of (weak) noncompactness on $X$. Let $A: D(A) \subseteq X \rightarrow X$ be a mapping. If $A(D(A))$ is bounded and for every nonempty bounded subset $M$ of $D(A)$ with $\psi(M)>0$, we have $\psi(A(M))<\psi(M)$; then $A$ is called $\psi$-condensing. If there exists $k, 0 \leq k \leq 1$, such that $A(D(A))$ is bounded and for each nonempty bounded subset $M$ of $D(A)$, we have $\psi(A(M)) \leq$ $k \psi(M)$; then $A$ is called $k$ - $\psi$-contractive.
Definition 4 (see [6]). A map $A: D(A) \rightarrow X$ is said to be ws-compact if it is continuous, and for any weakly convergent sequence $\left(x_{n}\right)_{n \in \mathbb{N}}$ in $D(A)$ the sequence $\left(A x_{n}\right)_{n \in \mathbb{N}}$ has a strongly convergent subsequence in $X$.

Definition 5. A map $A: D(A) \rightarrow X$ is said to be wwcompact if it is continuous, and for any weakly convergent sequence $\left(x_{n}\right)_{n \in \mathbb{N}}$ in $D(A)$ the sequence $\left(A x_{n}\right)_{n \in \mathbb{N}}$ has a weakly convergent subsequence in $X$.

Definition 6. Let $X$ be a Banach space. A mapping $T: D(T) \subseteq$ $X \rightarrow X$ is called a nonlinear contraction if there exists a continuous and nondecreasing function $\varphi: \mathbb{R}^{+} \rightarrow \mathbb{R}^{+}$such that

$$
\|T x-T y\| \leq \varphi(\|x-y\|),
$$

for all $x, y \in D(T)$, where $\varphi(r)<r$ for $r>0$.

In this paper we consider the semilinear equation

$$
A u+\lambda F(u)=f
$$

where $A: H \rightarrow H$ is a linear self-adjoint operator, satisfying a strong positivity condition, $F: H \rightarrow H$ is a nonlinear Lipschitz operator, and $\lambda$ is a positive parameter. Using the Banach fixed point theorem, we prove an existence and uniqueness result about the considered equation. Thus, we obtain here the same type of result as in [2], replacing the maximal monotonicity of linear part $A$ of the semilinear equation with the hypothesis that $A$ is self-adjoint. So, the principal result of this paper can be applied in the study of nonlinear Lipschitz perturbations of a linear integral operator with symmetric kernel. Further a result of continuous dependence on the free term and a fixed point theorem are presented. As applications we present some common fixed point theorems and approximation results for a pair of nonlinear mappings. Finally, the existence of solution of an integral equation is provided to illustrate the usability of the obtained results.

\section{Results}

Theorem 7. Let $A: H \rightarrow H$ be a linear self-adjoint operator and $F: H \rightarrow H$ nonlinear, satisfying the following conditions:

(i) F is a Lipschitz operator, that is, there is a constant $M>$ 0 such that

$$
\|F(x)-F(y)\| \leq M\|x-y\|,
$$

for all $x, y \in H$;

(ii) $A$ is a strongly positive operator, that is, there is a constant $c>0$ such that

$$
\langle A x, x\rangle \geq c\|x\|^{2},
$$

for all $x \in H$.

Then the equation $A u+\lambda F(u)=f$ has a unique solution for all $f \in H$ and $\lambda \in(0 ; c / M)$. 
Proof. Let us choose $s$ in the spectrum of $A$. We have

$$
s \geq \inf \{\langle A x, x\rangle \mid x \in H,\|x\|=1\} \geq c,
$$

and we obtain that every real number $\omega \in(-\infty ; c)$ is in the resolvent set of the operator $A$. Consequently, we have

$$
\operatorname{Rg}(A-\omega I)=H,
$$

for all $\omega<c$, where $I$ is the identity of $H$.

Let $\delta<0$. We write (9) in the equivalent form

$$
A_{\delta} u+F_{\delta}(u)=f \text {, }
$$

where $A_{\delta}=A-\delta I$ and $F_{\delta}=\lambda F+\delta I$. We have $\operatorname{Rg}\left(A_{\delta}\right)=H$ and

$$
\left\|F_{\delta}(x)-F_{\delta}(y)\right\| \leq(\lambda M+|\delta|)\|x-y\|,
$$

for all $x, y \in H$.

Also

$$
\begin{aligned}
\left\langle A_{\delta} x, x\right\rangle & =\langle A x, x\rangle-\delta\|x\|^{2} \\
& \geq(c-\delta)\|x\|^{2}=(c+|\delta|)\|x\|^{2} \quad \forall x \in H .
\end{aligned}
$$

From (16) we obtain

$$
\left\|A_{\delta} x\right\| \geq(c+|\delta|)\|x\| \quad \forall x \in H .
$$

Consequently, there exists $A_{\delta}^{-1}: H \rightarrow H$ which is linear and continuous, that is $A_{\delta}^{-1} \in \mathscr{L}(H)$, the Banach space of all linear and bounded operators from $H$ to $H$. Moreover, we have

$$
\left\|A_{\delta}^{-1}\right\|_{\mathscr{L}(H)} \leq \frac{1}{c+|\delta|} .
$$

Now (14) can be equivalently written as

$$
u+A_{\delta}^{-1} F_{\delta} u=A_{\delta}^{-1} f .
$$

We consider the operator $T: H \rightarrow H$ defined by

$$
T u=-A_{\delta}^{-1} F_{\delta} u+A_{\delta}^{-1} f .
$$

Therefore (19) becomes

$$
u=T u
$$

and so, the problem of the solvability of (9) is reduced to the study of fixed points of the operator $T$. We have

$$
\begin{aligned}
\|T x-T y\| & =\left\|A_{\delta}^{-1} F_{\delta} x-A_{\delta}^{-1} F_{\delta} y\right\| \\
& =\left\|A_{\delta}^{-1}\left(F_{\delta} x-F_{\delta} y\right)\right\| \leq\left\|A_{\delta}^{-1}\right\|_{\mathscr{L}(H)}\left\|F_{\delta} x-F_{\delta} y\right\| \\
& \leq \frac{\lambda M+|\delta|}{c+|\delta|}\|x-y\| \quad \forall x, y \in H .
\end{aligned}
$$

It results that $T$ is a strict contraction from $H$ to $H$ because $\lambda M<c$. According to the Banach fixed point theorem, $T$ has a unique fixed point, and thus the proof of Theorem 7 is complete.
Let us consider now the dependence of solution of (9) on the data $f$.

Theorem 8. Under the assumptions from the hypothesis of Theorem 7 , let $i \in\{1,2\}$, and let $u_{i}$ be the unique solution of the equation

$$
A u+\lambda F(u)=f_{i}, \quad i \in\{1,2\} ; f_{1}, f_{2} \in H .
$$

Then

$$
\left\|u_{1}-u_{2}\right\| \leq \frac{1}{c-\lambda M}\left\|f_{1}-f_{2}\right\|
$$

Proof. According to the equivalent form (19) of (9), we have

$$
\begin{aligned}
\left\|u_{1}-u_{2}\right\| & =\left\|-A_{\delta}^{-1} F_{\delta} u_{1}+A_{\delta}^{-1} f_{1}+A_{\delta}^{-1} F_{\delta} u_{2}-A_{\delta}^{-1} f_{2}\right\| \\
& \leq\left\|A_{\delta}^{-1} F_{\delta} u_{1}-A_{\delta}^{-1} F_{\delta} u_{2}\right\|+\left\|A_{\delta}^{-1} f_{1}-A_{\delta}^{-1} f_{2}\right\| \\
& \leq \frac{\lambda M+|\delta|}{c+|\delta|}\left\|u_{1}-u_{2}\right\|+\frac{1}{c+|\delta|}\left\|f_{1}-f_{2}\right\| .
\end{aligned}
$$

It results that

$$
(c-\lambda M)\left\|u_{1}-u_{2}\right\| \leq\left\|f_{1}-f_{2}\right\|,
$$

and thus our assertion is proved.

In fact, Theorem 8 establishes the continuous dependence of the solution of (9) on the free term and signifies the stability of the solution.

\section{Consequences of Principal Result}

The previous results prove that, under the considered assumptions, the operator $A+\lambda F$ is invertible for all $\lambda \epsilon$ $(0 ; c / M)$ and the inverse $C=(A+\lambda F)^{-1}$ is a Lipschitz operator. From (24) it results that the operator $C$ satisfies

$$
\left\|C\left(f_{1}\right)-C\left(f_{2}\right)\right\| \leq \frac{1}{c-\lambda M}\left\|f_{1}-f_{2}\right\| \quad \forall f_{1}, f_{2} \in H .
$$

Suppose now that $c>1$ and $\lambda \in(0 ;(c-1) / M) \subset(0 ; c / M)$. We obtain $M \lambda<c-1$ or $1 /(c-\lambda M)<1$, and, consequently, $C$ is a strict contraction. It results that there exists an unique element $u^{*} \in H$ such that $C\left(u^{*}\right)=u^{*}$ which is equivalent to $(A+\lambda F)\left(u^{*}\right)=u^{*}$. So we obtained the following fixed point theorem.

Theorem 9. Let $A: H \rightarrow H$ be a linear self-adjoint operator and $F: H \rightarrow H$ nonlinear, satisfying the following conditions:

(i) $F$ is a Lipschitz operator, that is, there is a constant $M>$ 0 such that

$$
\|F(x)-F(y)\| \leq M\|x-y\|,
$$

for all $x, y \in H$;

(ii) A is a strongly positive operator, that is, there is a constant $c>0$ such that

$$
\langle A x, x\rangle \geq c\|x\|^{2},
$$

for all $x \in H$. 
If $c>1$ and $\lambda \in(0 ;(c-1) / M)$, then the operator $A+\lambda F$ has a unique fixed point.

The way used in obtaining Theorem 9 can be applied in the study of the following problem: extracting operators which have the unique fixed point property from a family of operators $\left\{V_{\lambda} / \lambda \in \mathbf{R}\right\}$.

Let $E$ be a Banach space, and $\mathscr{F}=\left\{V_{\lambda}: E \rightarrow E / \lambda \in \mathbf{R}\right\}$, $V_{\lambda}$ satisfying some constraints for any $\lambda \in \mathbf{R}$. Our intention is to extract a subfamily $\mathscr{G} \subset \mathscr{F}$, so that $V_{\lambda}$ can have a unique fixed point for all $V_{\lambda} \in \mathscr{G}$. It is easy to observe, using the same method as in obtaining Theorem 9, that the following result holds.

\section{Theorem 10. If}

(i) there exists $J \subset \mathbf{R}, J \neq \phi$, so that $V_{\lambda}$ is invertible and

$$
\left\|V_{\lambda}^{-1} x-V_{\lambda}^{-1} y\right\| \leq g(\lambda)\|x-y\|, \quad x, y \in E ; g(\lambda)>0,
$$

$$
\text { for all } \lambda \in J \text {; }
$$

(ii) there exists $I \subset J, I \neq \phi$, so that $g(\lambda)<1$ for all $\lambda \in I$, then $V_{\lambda}$ has a unique fixed point for all $\lambda \in I$.

As an application of the observations established above, we develop here the Krasnoselskii and Ky Fan type approximation results for certain pair of maps.

Theorem 11. Let $A, F, \lambda, M$ and $c$ be as in Theorem $9, T=(A+$ $\lambda F)^{-1}$, and let $H$ be an ordered Hilbert space. Let $D$ be a nonempty closed convex subset of $H$ and $\psi$ a set additive measure of noncompactness on $H$. Let $S: H \rightarrow H$ be a mapping satisfying the following:

(i) $T(D) \subseteq D$ and $S(D) \subseteq D$,

(ii) $S$ is continuous and $\psi$-condensing,

(iii) $T$ and $S$ are weakly isotone.

Then there exists a unique $x^{*} \in D$ such that $A S x^{*}+$ $\lambda F S x^{*}=x^{*}$.

Proof. By Theorem 9, $T=(A+\lambda F)^{-1}: H \rightarrow H$ is a contraction with contractive constant $\alpha=1 /(c-\lambda M)<1$. Thus $T$ is a shrinking mapping. Now all of the conditions of Corollary 1.18 [7] are satisfied so there exists an $x^{*} \in D$ such that $T x^{*}=S x^{*}=x^{*}$ which implies that $(A+\lambda F) o S x^{*}=x^{*}$, and hence $A S x^{*}+\lambda F S x^{*}=x^{*}$.

Theorem 12. Let $A, F, \lambda, M$, and $c$ be as in Theorem 9, $T=$ $(A+\lambda F)^{-1}$, and let $H$ be an ordered Hilbert space. Let $D$ be a nonempty closed convex subset of $H$ and $\psi$ a set additive measure of noncompactness on $H$. Let $S: H \rightarrow H$ be a mapping satisfying the following:

(i) $T(D) \subseteq D$ and $S(D) \subseteq D$,

(ii) $S$ is a nonlinear contraction,

(iii) $T$ and $S$ are weakly isotone.
Then there exists a unique $x^{*} \in D$ such that $A S x^{*}+$ $\lambda F S x^{*}=x^{*}$.

Proof. By Theorem 9, $T=(A+\lambda F)^{-1}: H \rightarrow H$ is a contraction with contractive constant $\alpha=1 /(c-\lambda M)<1$. Thus $T$ is a shrinking mapping. Now all of the conditions of Corollary 1.19 [7] are satisfied so there exists an $x^{*} \in D$ such that $T x^{*}=$ $S x^{*}=x^{*}$ which implies $A S x^{*}+\lambda F S x^{*}=x^{*}$.

As an application of Corollaries 1.24 or 1.25 and 1.30 or 1.31 [7], we obtain the following results, respectively

Theorem 13. Let $A, F, \lambda, M$, and $c$ be as in Theorem 9, $T=$ $(A+\lambda F)^{-1}$, and let $H$ be an ordered Hilbert space. Let $D$ be a nonempty closed convex subset of $H$ and $\psi$ a set additive measure of weak noncompactness on $H$. Assume that $S, T: H \rightarrow H$ are sequentially weakly continuous mappings satisfying the following:

(i) $T(D) \subseteq D$ and $S(D) \subseteq D$,

(ii) $S$ is $\psi$-condensing or $S$ is a nonlinear contraction,

(iii) $T$ and $S$ are weakly isotone.

Then there exists a unique point $x^{*} \in D$ such that $A S x^{*}+$ $\lambda F S x^{*}=x^{*}$.

Theorem 14. Let $A, F, \lambda, M$, and $c$ be as in Theorem 9, $T=$ $(A+\lambda F)^{-1}$, and let $H$ be an ordered Hilbert space. Let $D$ be a nonempty closed convex subset of $H$ and $\psi$ a set additive measure of weak noncompactness on $H$. Assume that $S, T$ satisfy the following:

(i) $T$ is a ww-compact mapping,

(ii) $S$ is continuous ws-compact and $\psi$-condensing or $S$ is continuous ws-compact and nonlinear contraction,

(iii) $T$ and $S$ are weakly isotone,

(iv) $T(D) \subseteq D$ and $S(D) \subseteq D$.

Then there exists a unique $x^{*} \in D$ such that $A S x^{*}+$ $\lambda F S x^{*}=x^{*}$.

Theorem 15. Let $H, A, F, \lambda, M$, and $c$ be as in Theorem 9 and $T=(A+\lambda F)^{-1}$ sequentially weakly continuous. Assume that $D$ is nonempty closed bounded convex subset of $H$ and $S: D \rightarrow H$ is sequentially weakly continuous mapping satisfying the following:

(i) $S(D)$ is relatively weakly compact,

(ii) $(x=T x+S y ; y \in D) \Rightarrow x \in D$.

Then there exists a unique point $x^{*} \in D$ such that $(A+$ $\lambda F) o(I-S) x^{*}=x^{*}$.

Proof. By Theorem 9, $T=(A+\lambda F)^{-1}: H \rightarrow H$ is a strict contraction with contractive constant $\alpha=1 /(c-\lambda M)<1$. Now Theorem 2.1 [6] implies that there exists an $x^{*} \in D$ such that $S x^{*}+T x^{*}=x^{*}$ which implies that $(A+\lambda F) o(I-S) x^{*}=$ $x^{*}$. 
Theorem 16. Let $A, F, \lambda, M$, and $c$ be as in Theorem 9, $T=$ $(A+\lambda F)^{-1}$, and let $H$ be an ordered Hilbert space. Let $D$ be a nonempty closed convex subset of $H$ and $\psi$ a set additive measure of noncompactness on $H$. Let $S: H \rightarrow H$ be a mapping satisfying the following:

(i) $S(D) \subseteq D$,

(ii) $S$ is continuous and $\psi$-condensing or ( $S$ is a nonlinear contraction),

(iii) PT and $S$ are weakly isotone,

where $P$ is the proximity map on $D$. Then there exists $x_{0} \in D$ such that

$$
\begin{aligned}
\left\|x_{0}-T x_{0}\right\| & =\left\|S x_{0}-T x_{0}\right\| \\
& =d\left(T x_{0}, D\right)=d\left(T x_{0}, \overline{I_{D}\left(x_{0}\right)}\right) .
\end{aligned}
$$

More precisely, either

(1) $S$ and $T$ have a common fixed point $x_{0} \in D$, or

(2) there exists $x_{0} \in \partial D$ with

$$
\begin{aligned}
0 & <\left\|T x_{0}-S x_{0}\right\|=\left\|T x_{0}-x_{0}\right\| \\
& =d\left(T x_{0}, D\right)=d\left(T x_{0}, \overline{I_{D}\left(x_{0}\right)}\right) .
\end{aligned}
$$

Proof. Let $P$ be the proximity map on $D$; that is, for each $x \in$ $H$, we have $\|P x-x\|=d(x, D)$. It is well known that $P$ is nonexpansive in $H$. As $T$ is shrinking map, so $P T: D \rightarrow D$ is also shrinking mapping. By Theorem 4.1 (or 4.2), there exists $x_{0} \in$ $M$ such that $x_{0}=S x_{0}=P T x_{0}$. Thus we obtain, as in Theorem $4.1[8]$ the desired conclusion.

Following the proof of Corollary 4.5 [8] and using Theorem 16, we obtain the following common fixed point theorem.

Theorem 17. Let $A, F, \lambda, M$, and $c$ be as in Theorem 9, $T=$ $(A+\lambda F)^{-1}$, and let $H$ be an ordered Hilbert space. Let $D$ be a nonempty closed convex subset of $H$ and $\psi$ a set additive measure of noncompactness on $H$. Assume that $S: H \rightarrow H$ is a mapping satisfying the following:

(i) $S(D) \subseteq D$,

(ii) $S$ is continuous and $\psi$-condensing or ( $S$ is a nonlinear contraction),

(iii) PT and S are weakly isotone,

where $P$ is the proximity map on D. Suppose that $T$ satisfies one of the following conditions for each $x \in \partial D$, with $x \neq T x$ :

(i) $\|T x-y\|<\|T x-x\|$ for some $y$ in $\overline{I_{D}(x)}$;

(ii) there is a $\gamma$ such that $|\gamma|<1$ and $\gamma x+(1-\gamma) T x \in \overline{I_{D}(x)}$;

(iii) $T x \in \overline{I_{D}(x)}$;

(iv) for each $\gamma \in(0,1), x \neq \gamma T x$;

(v) there exists an $\alpha \in(1, \infty)$ such that, $\|T x-x\|^{\alpha} \geq$ $\|T x\|^{\alpha}-r^{\alpha}$

(vi) there exists $\beta \in(0,1)$ such that, $\|T x-x\|^{\beta} \leq\|T x\|^{\beta}-$ $r^{\beta}$.

Then $F(S) \cap F(T) \neq \emptyset$.

\section{An Application}

Fixed point theorems for certain operators have found various applications in differential and integral equations (see [7-10] and references therein). In this section, we present an application of our Theorem 7 to establish a solution of a nonlinear integral equation.

Let $K:[0,1] \times[0,1] \rightarrow \mathbf{R}$ be a continuous function, and suppose that $K$ is symmetric (i.e., $K(x, y)=K(y, x)$ for all $x, y \in[0,1])$. We consider the linear operator $B: L_{\mathbf{R}}^{2}[0,1] \rightarrow$ $L_{\mathbf{R}}^{2}[0,1]$ defined by $B u(t)=\int_{0}^{1} K(t, s) u(s) d s$. It is easy to observe that $B$ is a self-adjoint operator. Now let $A$ : $L_{\mathbf{R}}^{2}[0,1] \rightarrow L_{\mathbf{R}}^{2}[0,1]$ defined by $A u(t)=u(t)+\iint_{0}^{1} K(t, s)$ $K(s, z) u(z) d z d s$; that is, $A=I+B^{2}$ where $I$ is the identity of $L_{\mathbf{R}}^{2}[0,1] . A$ is a self-adjoint strongly positive operator satisfying $\langle A u, u\rangle_{2}=\langle u, u\rangle_{2}+\left\langle B^{2} u, u\right\rangle_{2} \geq\|u\|_{2}^{2}\left(\langle\cdot, \cdot\rangle_{2}\right.$ and $\|\cdot\|_{2}$ signify the inner product and the norm in $\left.L_{\mathbf{R}}^{2}[0,1]\right)$.

Define $f:[0,1] \times \mathbf{R} \rightarrow \mathbf{R},(t, u) \mapsto f(t, u)$, having partial derivative of first order in the second variable $u$ and $|(\partial f / \partial u)(t, u)| \leq M$ for all $(t, u) \in[0,1] \times \mathbf{R}(M>0)$. $F: L_{\mathbf{R}}^{2}[0,1] \rightarrow L_{\mathbf{R}}^{2}[0,1]$ defined by $F u(t)=f(t, u(t))$ is a Lipschitz operator of constant $M$ from $L_{\mathbf{R}}^{2}[0,1]$ into $L_{\mathbf{R}}^{2}[0,1]$.

Define nonlinear integral equation

$u(t)+\int_{0}^{1} \int_{0}^{1} K(t, s) K(s, z) u(z) d z d s+\lambda f(t, u(t))=g(t)$,

$g \in L_{\mathbf{R}}^{2}[0,1]$

where $\lambda \in(0 ; 1 / M)$. All of the conditions of Theorem 7 are satisfied, so the above-mentioned integral equation has a unique solution for all $\lambda \in(0 ; 1 / M)$. Moreover, by Theorem 8 the solution depends continuously on the free term $g$.

\section{Acknowledgment}

The work of first author was supported by a grant of the Romanian National Authority for Scientific Research, CNCSUEFISCDI, project number PN-II-ID-PCE-2011-3-0087.

\section{References}

[1] H. Amann, "On the unique solvability of semilinear operator equations in Hilbert spaces," Journal de Mathématiques Pures et Appliquées, vol. 61, no. 2, pp. 149-175, 1982.

[2] H. Amann, "Saddle points and multiple solutions of differential equations," Mathematische Zeitschrift, vol. 169, no. 2, pp. 127$166,1979$.

[3] D. Teodorescu, "A contractive method for a semilinear equation in Hilbert spaces," Analele Universitatii Bucuresti Matematica, vol. 54, no. 2, pp. 289-292, 2005.

[4] J. Banaś and J. Rivero, "On measures of weak noncompactness," Annali di Matematica Pura ed Applicata, vol. 151, pp. 213-224, 1988.

[5] F. S. de Blasi, "On a property of the unit sphere in a Banach space," Bulletin Mathématique de la Société des Sciences Mathématiques de Roumanie, vol. 21, no. 3-4, pp. 259-262, 1977. 
[6] M. A. Taoudi, "Krasnosel'skii type fixed point theorems under weak topology features," Nonlinear Analysis: Theory, Methods \& Applications, vol. 72, no. 1, pp. 478-482, 2010.

[7] R. P. Agarwal, N. Hussain, and M.-A. Taoudi, "Fixed point theorems in ordered Banach spaces and applications to nonlinear integral equations," Abstract and Applied Analysis, vol. 2012, Article ID 245872, 15 pages, 2012.

[8] N. Hussain, A. R. Khan, and R. P. Agarwal, "Krasnosel'skii and Ky Fan type fixed point theorems in ordered Banach spaces," Journal of Nonlinear and Convex Analysis, vol. 11, no. 3, pp. 475489, 2010.

[9] N. Hussain and M. A. Taoudi, "Krasnosel'skii-type fixed point theorems with applications to Volterra integral equations," Fixed Point Theory and Applications, vol. 2013, article 196, 2013.

[10] K. Latrach, M. A. Taoudi, and A. Zeghal, "Some fixed point theorems of the Schauder and the Krasnosel'skii type and application to nonlinear transport equations," Journal of Differential Equations, vol. 221, no. 1, pp. 256-271, 2006. 


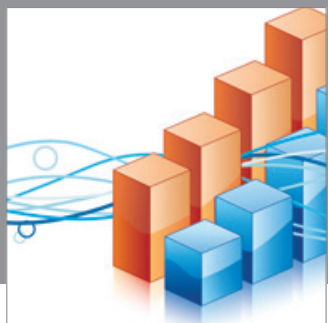

Advances in

Operations Research

mansans

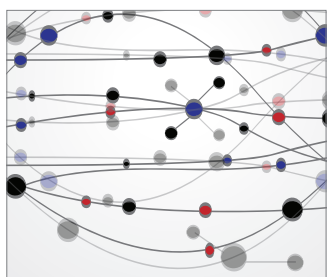

The Scientific World Journal
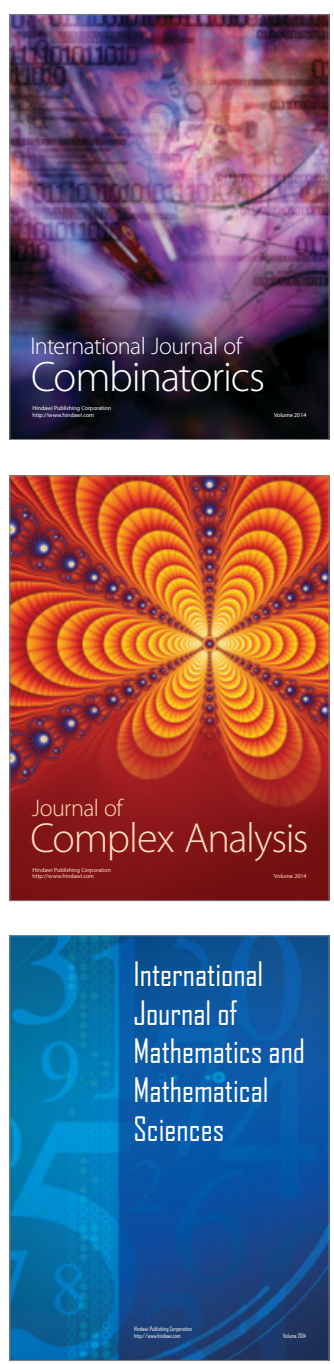
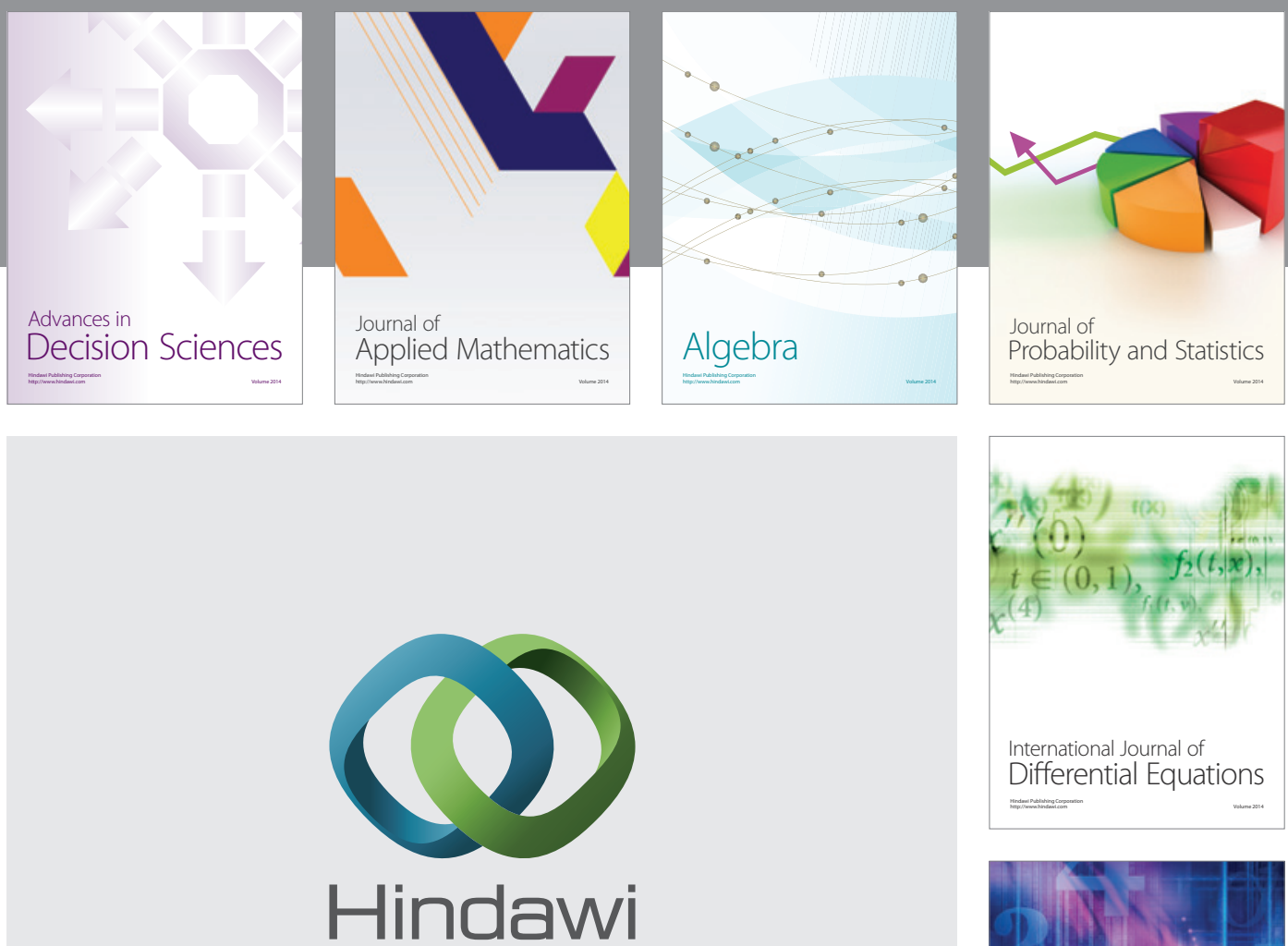

Submit your manuscripts at http://www.hindawi.com
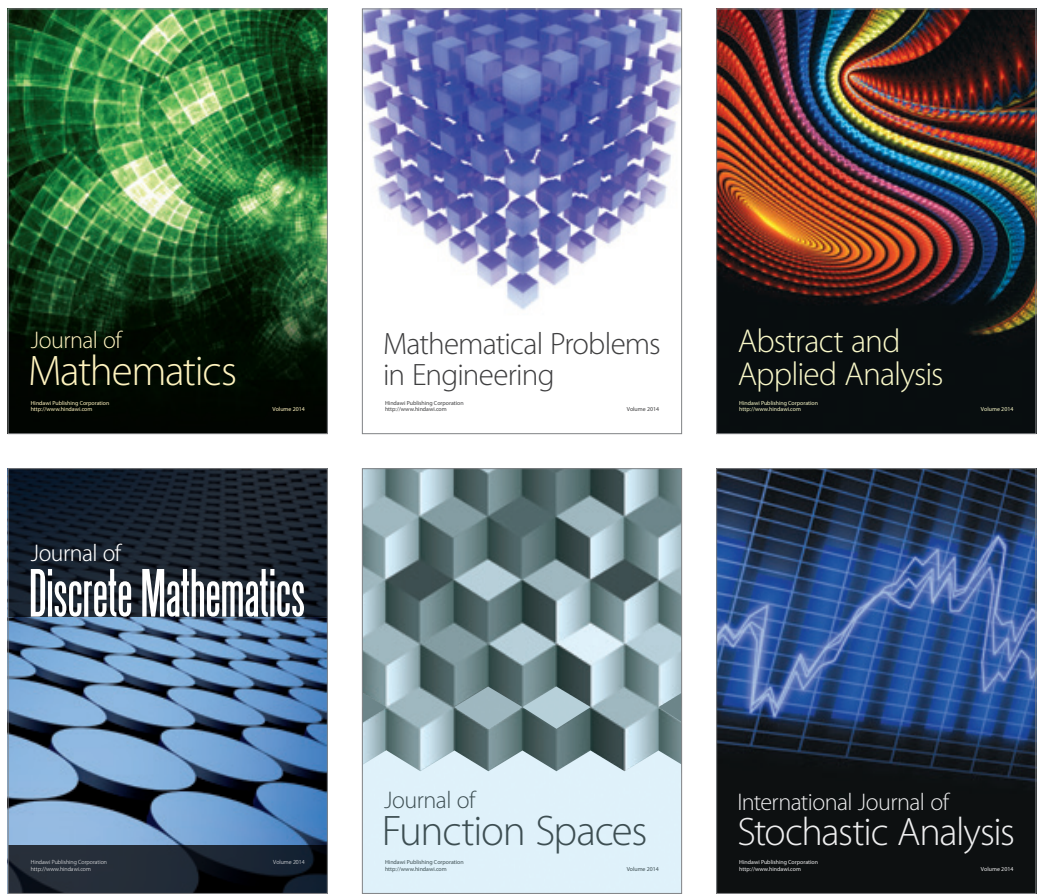

Journal of

Function Spaces

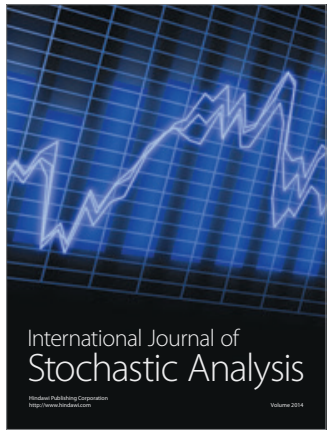

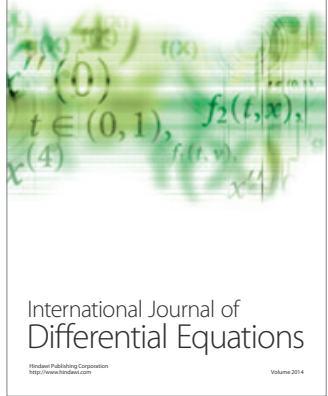
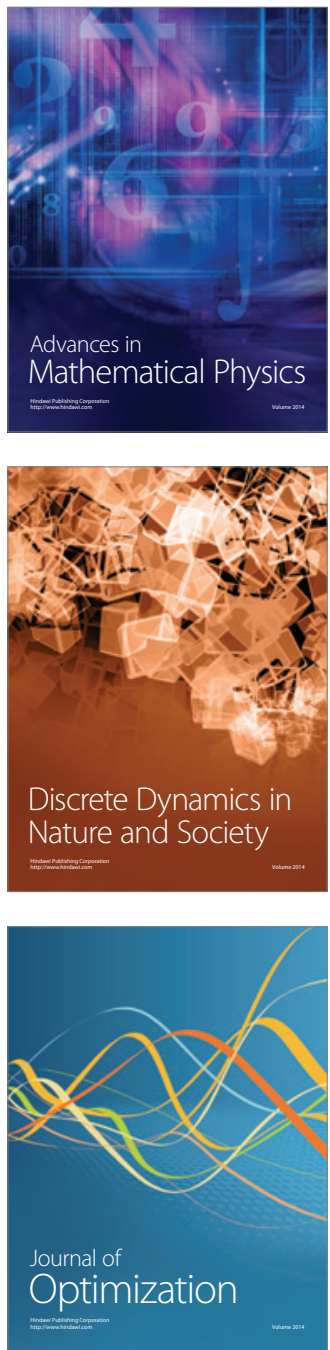